\title{
CHAAJ (JUEGO DE PELOTA MESOAMERICANO): UN JUEGO ANCESTRAL ENTRE EMERGENCIAS CULTURALES
}

\author{
CHAAJ (MESO-AMERICAN BALL GAME): \\ AN ANCESTRAL GAME AMONG CULTURAL EMERGENCIES
}

Jairzinho Francisco Panqueba Cifuentes ${ }^{1}$

Resumen

Los juegos de pelota mesoamericanos son manifestaciones corporales que han sido exploradas principalmente desde perspectivas arqueológicas e históricas, pero también han sido retomados desde distintas iniciativas para ponerlos en práctica. Desde la frontera entre Estados Unidos con México, pasando por distintos Estados del país "azteca", son practicadas diferentes modalidades de este juego. Sin embargo, decir que el chaaj en Mesoamérica es hoy en día una alternativa recreativa y deportiva, es quedarse corto respecto a su ya demostrado potencial. La sacralidad manifestada a través de los movimientos corporales está ofreciendo opciones de innovación en varios espacios de las sociedades actuales. En su dimensión ceremonial, revela una comunicación ancestral muy actual. Allí se ponen en juego los códices, las interpretaciones arqueológicas y los conocimientos territoriales de personas sabedoras de las comunidades. En su dimensión lúdica, el juego reúne elementos culturales, deportivos y pedagógicos. Ha sido una práctica corporal, técnica y motora ejecutada constantemente en algunas regiones mexicanas y guatemaltecas. No obstante su antigüedad, en los últimos años se viene registrando una promoción inusitada, en medio de los actuales tiempos de cambio que fueron anunciados desde tiempos inmemoriales por los sabedores y las sabedoras mayas.

Palabras clave: chaaj, pelota, mayas, Guatemala, lúdica, ancestral

Abstract

Meso-American ball games are corporal manifestations that have been explored mainly from archaeological and historical perspectives. They have also been revisited from different initiatives that seek to put them into practice. From the frontier among United States with Mexico, going through different States of the "Aztec" country, different modalities of this game are being practiced. However, to say that the chaaj in Mesoamerica is today a recreational and sport alternative, is insufficient regarding its demonstrated potential. The sacredness manifested through corporal movements offers options for innovation in several spaces of current societies. In their ceremonial dimension, it reveals a very current and effective ancestral communication. There, the codices, the archaeological interpretations and the territorial knowledge of knowing people of the communities are put at stake. In their recreational dimension, the game gathers cultural, sport and pedagogic elements. It has been a corporal practice and technique, constantly practiced in some Mexican and Guatemalan regions. Despite their validity, an unusual promotion has been occurring during recent years, in the current changing times that were announced since immemorial times by the Mayan wise men a women.

Keywords: chaaj, ball, mayas, Guatemala, recreational, ancient.

Fecha de recepción: 8 de julio de 2011

Fecha de aprobación: 8 de octubre de 2011

1 Aprehendedor de saberes ancestrales y de la vida cotidiana. Doctor en Ciencias Sociales con especialidad en Antropología Social del Centro de Investigaciones y Estudios Superiores en Antropología Social (CIESAS)-Guadalajara, Jalisco, México. Magister en Ciencias Sociales con énfasis en Estudios Étnicos de la Facultad Latinoamericana de Ciencias Sociales (FLACSO)- Ecuador. Licenciado en Educación Física de la Universidad Pedagógica Nacional, Bogotá, Colombia. Docente del Colegio San Bernardino IED, territorio muisca de Bosa. Correo-E: panqueba@gmail.com 


\section{Territorios de un juego sagrado}

Este artículo revisa algunos resultados preliminares de una investigación que aborda los juegos ancestrales, desde iniciativas que propenden por su revitalización y práctica. Para la indagación decidimos tomar dos casos: el zepcuagoscua ${ }^{2}$, popularmente conocido como tejo o turmequé de los territorios chibchas ${ }^{3}$ de Colombia, y el chaaj de los territorios mayas de Guatemala. Hemos buscado los senderos del fortalecimiento de la práctica del chaaj como un espacio de formación espiritual para las generaciones jóvenes guatemaltecas. Por otra parte, hemos abordado desde los aportes mayas, los elementos ceremoniales previos, in situ y posteriores que se efectúan en el juego del zepcuagoscua en Colombia. Sin embargo, en este escrito sólo damos cuenta del chaaj, porque nos ha permitido analizar el inusitado interés que desde hace dos décadas han despertado algunas de las prácticas ancestrales de los pueblos indígenas.

La vistosidad, el performance y las habilidades corporales exhibidas durante los juegos ancestrales contrastan con la utilización que cada población, institución y actores han dado a los mismos. Si bien actualmente existe un conocimiento básico sobre las distintas manifestaciones culturales de los pueblos, los juegos ancestrales indígenas en los últimos tiempos han representado un espectáculo étnico muy ajeno a su origen mismo. Ello ha sucedido por la reproducción de los poderes y colonizaciones que continúan retomando ciertas expresiones culturales específicas de los pueblos, para mostrarlas como eje de identidad nacional y como espectáculo esencialista, algo muy lejano de lo que estas manifestaciones realmente son para cada pueblo. Por estas razones, hemos pretendido investigar e intervenir la sostenibilidad de iniciativas que apunten hacia la inclusión de las prácticas ancestrales en lo contemporáneo, manteniendo sus contenidos sacros y pedagogías abyayalenses ${ }^{4}$.

2 Esta expresión es en idioma chibcha de Colombia, que traducida al castellano significa la acción de jugar un juego de entretenimiento.

3 La familia lingüística chibcha es la que más pueblos indígenas abarca en Colombia. Sus poblaciones están ubicadas principalmente en la cordillera de los Andes, a lo largo de los departamentos de Cundinamarca, Boyacá, Santander y Norte de Santander. Los pueblos de la Sierra Nevada de Santa Marta (Arhuaco, Kogui y Arsario) también hacen parte de esta matriz lingüística.

4 La expresión abya-yalense deriva de Abya- Yala, palabra con la cual es denominado el continente americano en idioma de los Kuna, pueblo ancestral que habita en territorios de Colombia y Panamá. Abya-Yala también es la palabra que los
En este camino de indagación, nos hemos permitido contemplar las aproximaciones que las actuales generaciones mayas han realizado a su juego ancestral. Pero también hemos podido confrontar estas actividades con los usos del juego que desde hace algunas décadas han estado realizando distintos actores. Nos referimos específicamente a la organización de campeonatos desde una perspectiva deportiva, así como a la utilización del juego como atractivo exótico para el turismo nacional e internacional, que cada año visita los centros arqueológicos y territorios mayas. Estos performances convergen con el dinamismo de las culturas americanas, anunciado desde los tiempos inmemoriales por los sabedores y las sabedoras mayas. Pero también representan el riesgo de instrumentalizar el juego, haciéndolo sucumbir con su transformación como una sencilla práctica deportiva, de espectáculo o como folclor.

A continuación describo un recorrido de aprendizajes que hemos compartido con jugadores de pelota, nacionales guatemaltecos y mayas de origen (Panqueba, 2010), que laboran para el Ministerio de Cultura y Deportes de Guatemala -MICUDE- en un programa llamado Juego de pelota Maya (MICUDE, 2011). Éste se ha desarrollado desde el año 2002, inicialmente bajo la coordinación del investigador Manuel Eduardo Takatik Esquit (Ver MICUDE, 2008), y, desde el año 2010, por el compañero originario del pueblo Maya-Achí Jorge Mario López Tista. En las siguientes páginas comparto algunos recorridos realizados, al tiempo que voy entrelazando símbolos, descripción del juego y sus implicaciones en el entramado de emergencias culturales que han venido surgiendo durante la primera década del siglo XXI.

En primer lugar presento el contexto del juego, para describir someramente sus elementos y ejecución, desde la perspectiva de quienes dinamizan el chaaj como una manifestación lúdica ancestral y sagrada. En segundo lugar, delimito el campo de debate sobre las emergencias culturales frente a las cuales se hallan los juegos ancestrales. En tercer lugar, propongo algunos desarrollos analíticos sobre los juegos ancestrales frente a los desiguales usos de las emergencias culturales. Y en cuarto lugar, propongo unas conclusiones, considerando las posibilidades del juego de pelota maya en Guatemala.

\section{Invocaciones y evocaciones ancestrales}

movimientos y organizaciones indígenas han apropiado para referirse a los territorios invadidos por Europa desde finales del siglo XV y hasta ahora, a través de las interminables acciones de colonización. 
Durante la última década, varios grupos organizados de diversas formas, han estado dinamizando la práctica del chaaj en Guatemala. Entre estos encontramos la organización AJj zuk, un grupo de jóvenes quienes han realizado jornadas ecológicas y deportivas, a través de las cuales han difundido el chaaj. Refieren su actividad en torno al juego de pelota maya de la siguiente manera:

Tomando en cuenta el valor histórico, cultural y cosmogónico de este deporte, el movimiento juvenil tiene como deporte oficial el Chaaj, actualmente cuenta con 400 grupos en capacitación constante de los cuales se seleccionarán 100 equipos para el próximo campeonato nacional. Dentro del movimiento se practica de forma deportiva y competitiva entre equipos femeninos y masculinos de 8 integrantes por equipo. Nuestro objetivo es promover la actividad física, fortalecer nuestra cultura y rescatar este deporte ancestral (Aj Tzuk, 2011).

Por otra parte, desde la Dirección General de Educación Física-DIGEF- se han puesto en la tarea de estudiar e ir conformando un reglamento que permita hacer chaaj en las escuelas. Ello también mediante la formación de quienes estudian la carrera de educación física. Durante el año 2010, la DIGEF publicó un libro que recopila gran parte de historias y revisa investigaciones sobre el chaaj (DIGEF, 2010). También la DIGEF propone allí un reglamento y algunas alternativas pedagógicas que permitirían difundir en el ámbito escolar este juego.

En el MICUDE, el grupo de pelota maya realiza una labor promocional del juego dentro y fuera del país. Viaja a distintos lugares, llevando el juego a través de una exhibición que inicia con una ceremonia de invocación, dentro de la cual el coordinador del grupo explica los antecedentes ancestrales, históricos y arqueológicos del juego y proporciona información acerca de la forma de jugar, la puntuación y otros aspectos técnicos. Las indumentarias, pinturas corporales y presentación de cada jugador también hacen parte de las palabras compartidas con las personas asistentes.

Dado que el Ministerio cuenta con funcionarios en cada departamento y varios municipios, el grupo constantemente es convocado a presentarse. Cada semana pueden visitar por lo menos tres municipios. Allí preparan escenarios como coliseos, parques principales, calles, campos deportivos y otros, que facilitan la convocatoria de niñas, niños, jóvenes y población en general. El grupo viaja en un vehículo tipo pick-up, en el cual acarrean los componentes para armar y desarmar, en cuestión de minutos, una estructura o frontón de madera en cada lugar. Terminada la presentación, las seis tablas de $1 \mathrm{~m}$ x $2 \mathrm{~m}$ son desmontadas, y el armazón de tubos metálicos es convertido en una carga acomodada en la pick-up hasta otra nueva presentación del grupo.

El chaaj, nombre con el que se le conocía al juego de pelota durante la época postclásica, tiene su punto de partida en el descenso que hacen los hermanos Hunahpu e Ixbalanque hacia Xibalbá -el inframundo o infierno en la cosmovisión maya- luego de la invitación que los Ajaw -dioses- de Xibalbá les hacen:

Que vengan aquí a jugar a la pelota con nosotros, para que con ellos se alegren nuestras caras, porque verdaderamente nos causan admiración. Así pues vengan, dijeron los señores. Y que traigan acá sus instrumentos de juego, sus anillos, sus guantes, y que traigan también sus pelotas de caucho (Recinos, 2000, citado en DIGEF, 2010: 2) ${ }^{5}$.

Ese descenso hacia Xibalbá se empieza a sentir en los jugadores del Ministerio de Cultura cuando se colocan su atuendo, conformado por los siguientes elementos: Washot, Bate, Tz'um, Yachax, Pachq'ab (DIGEF, 2010: 92). Washot es un tocado para la cabeza, el cual sirve para recoger el cabello; Bate es un cinto elaborado en cuero, el cual se usa como protector para la cintura; Tz'um es una faldilla que se coloca atada en la cintura; Yachax es un tocado de cuero con una efigie que protege la zona genital del jugador; Pachq'ab es un protector elaborado en cuero, el cual cubre la mano y el antebrazo del jugador hasta la altura del codo. Además de estos instrumentos se usa una rodillera que, además de proteger, sirve para golpear la pelota. También hay unos adornos que se colocan en los brazos, muñecas, muslos y sobre los tobillos. En los pies usan unas sandalias o caites elaborados en cuero. La re-creación de este traje ha sido resultado de un trabajo de investigación arduo desde el MICUDE.

Para afrontar cada salida al campo de juego, finalizan la preparación del atuendo dibujando sobre el cuerpo de cada jugador distintas figuras. La cara, los brazos, el torax, la espalda y los hombros son cuidadosamente pintados con unos tintes especiales de colores rojo, blanco, negro, verde, azul y algunas mezclas resultantes entre estos. Las figuras plasmadas en la piel dependen del personaje que cada jugador representa en el campo de juego: Los ajaw de Xibalbá, Hunahpu e Ixbalanque.

Los atavíos que porta cada jugador preparan el ambiente ceremonial, demarcando la diferencia entre el mundo

5 Ver también: $\overline{\text { Arte Maya Naif (2011) }}$ 
cotidiano y el mundo del chaaj. Un mundo al que se puede entrar con una transformación personal y espacial. En este sentido, cabe recordar que los objetos transicionales funcionan como mediadores entre el entorno real y el simbólico (Weisz, 1993:71). Cada uno de los jugadores, mediante los elementos del traje y el maquillaje corporal, inicia su propio descenso hacia Xibalba. Al seguir los pasos rituales y ubicarse en el campo de juego, el jugador habita la realidad subjetiva que los ancestros y las ancestras han explorado en los tiempos inmemoriales (DIGEF, 2010: 72). "El cuerpo del jugador debe sacralizarse para entrar al terreno de juego, ya que en su persona se realizará el drama divino del origen" (DIGEF, 2010: 73).

Luego de la sacralización personal, los jugadores disponen los elementos para realizar un ritual de inicio. De esta manera, unas vasijas de arcilla que contienen ocote $^{6}$, copal $^{7}$ y algunas piezas de carbón, son portadoras del fuego para ofrendar. Luego hacen sonar unos caracoles al viento para cerrar la sacralización del espacio. Presentan un componente escénico, el cual parece una representación de una danza o de un sketch teatral. Hay un momento en que cada jugador ejecuta libremente un movimiento que le caracteriza, a la vez que pronuncia una invitación en su respectivo idioma maya. Luego de ello, cada jugador permanece inmóvil unos cuantos segundos. Generalmente representan deidades mitológicas de animales mesoamericanos.

Aunque los juegos ancestrales pueden ser percibidos como show, deporte o espectáculo para turistas, lo que realmente sucede es la evocación de un juego sacro. Una manifestación que "(...) toma su ordenamiento por conducto de diferentes disposiciones rituales que deben cumplirse para restaurar un equilibrio cósmico o invocar la acción divina en el desarrollo del juego mismo" (Weisz, 1993: 10). En esta parte del juego sucede una conversación en la que se intercambian frases en idiomas mayas. Éstos son diálogos y representaciones que evocan escenas del Popol Vuh ${ }^{8}$, libro sagrado de

6 Trozos pequeños de madera de pino triste, árbol maderable común en Mesoamérica.

7 Incienso obtenido de una goma resinosa de varios árboles. Se usa en las ceremonias sagradas de los pueblos amerindios, así como en los sahumerios durante las celebraciones católicas. 8 Vocablo del idioma maya-quiché, que traducido al castellano significa Libro de la comunidad o libro del consejo. Popol = Reunión, junta, comunidad. Vuh= Libro. Es inexacta la información acerca de la autoría el libro. Según Francisco Jimenez, sacerdote católico que tradujo al castellano la primera versión escrita en idioma maya-quiché hacia el año 1701, el libro fue compilado a mediados del siglo XVI. Él conoció esta ver- los pueblos mayas que expone una serie de relatos de la cosmovisión Maya Quiché, también conocido como Popol Wuj (Saravia, 1969) o Pop Wuj (Chavez, 2010). Esta compilación trata sobre la creación del mundo, la religión e historias del pueblo quiché. En el intercambio de frases entre los jugadores se evoca un relato de esta obra, en el cual los hermanos Hunahpu e Ixbalanque, son invitados a jugar por parte de los señores de Xibalbá -inframundo- (Códice Trocortesiano, XXVIII y VI, en: Saravia, 1969: 86, 88).

El juego propiamente dicho se efectúa contra el tablero, frontón o muro de madera. Este elemento consta de un cuerpo desarmable, conformado por seis piezas de madera y soportes metálicos en la parte posterior. Mide aproximadamente cinco metros de largo por tres de alto. En la parte superior que divide el tablero en dos, se dispone un aro de metal sujetado a la estructura. Uno de los objetivos del juego es hacer pasar la pelota a través de dicho aro, que es golpeada con las partes autorizadas. De esa forma, se obtienen los tantos o rayas. En caso de contacto de la pelota con el pie, la mano o la cabeza, se restan rayas.

La primera jugada en el chaaj consiste en un lanzamiento de la pelota al suelo para que rebote en el campo opuesto. Allí la recibe otro jugador, que regularmente dirige su golpe hacia el frontón. Luego se realizan diferentes golpes a la pelota con la rodilla, la cintura y el antebrazo. En la modalidad llamada "de antebrazo", la pelota también se puede tocar con otras partes del cuerpo, a excepción de manos, cabeza o pies ${ }^{9}$. De acuerdo con el avance del marcador, se va re-acomodando la duración del encuentro. Ello en medio de retos y contra-retos que se intercambian efusivamente al tiempo que la pelota está en movimiento. Las frases son expresadas en los idiomas mayas que cada jugador domina.

El componente lúdico referente a los juegos con las palabras ha sido analizado por Huizinga (1987) como la necesaria lucha agonal por el honor. Cada jugador lanza porfías a sus contrarios, siempre buscando el sostenimiento del combate en un plano lingüístico; el juego transcurre en porfías nobles (Cfr. Huizinga, Op.cit.: 123). Para Weisz, "El poder rítmico y mágico de las palabras y movimientos le confiere un valor sacralizado a la cancha. Este concepto se añade a la pelota

sión gracias a que le fue compartida por la población de la localidad hoy llamada Chichicastenango, departamento del Quiché, Guatemala (Bibliografía en Saravia, 2004: LII, LIII). 9 Otros grupos de pelota juegan la modalidad de cintura. Allí sólo se protegen especialmente esta parte del cuerpo con un cinturón grueso, elaborado en cuero u otros materiales que puedan amortiguar un poco cada golpe. 
como movimiento cósmico y esencia de la fertilidad terrestre" (Weisz, Op.cit.: 80). Es la lucha entre opuestos complementarios en la escena lúdica. Para el juego de pelota mesoamericano, es la disputa entre día y noche, claridad y oscuridad; la pugna entre el sol y la luna.

\section{Jugar un juego en tiempos de emergencias cul- turales}

En un blog dedicado a resaltar aspectos ambientales, culturales y sociales de la República de Guatemala, el autor publicó una entrada con el título: "un juego que no era juego" (Mendizabal, 2010). Ello, para dar una idea de las dimensiones sacras del chaaj por su connotación ritual. Este componente crucial del juego, al ser exhibido durante actos en escuelas, plazas públicas, campos deportivos o en una explanada cubierta de grama, conduce a una dimensión cultural tal vez obviada en muchos deportes habitualmente practicados en la actualidad. Una dimensión que causa reacciones insospechadas entre las personas asistentes. Durante una de estas jornadas un asistente preguntaba: “ ¿esos son los mayas verdaderos?”. El asombro radicaba en la supuesta inexistencia actual de los mayas. Luego, quien preguntaba especulaba que seguramente habrían sacado a estos jugadores de una de "las ruinas" del sitio arqueológico en que estaban realizando la presentación.

Escuchamos estos y otros comentarios, desde los que elogian respetuosamente, pasando por los chistosos y burlones, hasta los que elogian sin comprender lo visto. Y es que el inusitado volcamiento de re-producciones ancestrales durante actos públicos ha abundado durante los últimos tiempos. Ello motiva un análisis a la luz de los escenarios que en la actualidad tienen los llamados procesos de re-creación, revitalización, recuperación, dinamización y demás relacionados con la preocupación por mantener la cultura.

El florecimiento de las variadas posibilidades para el chaaj se ocurre en un contexto favorable (proclive) a la promoción de expresiones étnicas en el mundo. Un ambiente en el que han convergido disímiles cambios para la humanidad. Para el caso de los pueblos indígenas, el suceso que marcó la entrada en vigor de este ambiente proclive al etnicismo, fue el que vivimos durante las conmemoraciones del año 1992. Que aunque fue la fecha de los festejos por el cumplimiento de los 500 años de la invasión europea en América, también originó una inusitada visibilización globalizada de los movimientos indígenas.
Posterior al fenómeno de las celebraciones de los 500 años del "descubrimiento de América", muchas personas y organizaciones re-significaron diferentes manifestaciones étnicas y las colocaron en el actual mercado del esoterismo, el neo-shamanismo, el new age y las tendencias ecologistas. Estos procesos de etnogénesis, discursos híbridos, etnoecologismos, ecoetnicidades y panindigenismos jugaron a favor de las reinvenciones identitarias indígenas, pero también de quienes lucran contra las mismas. Guatemala es un país que ha vivido este proceso durante los últimos veinte años, el cual se ha venido analizando con el concepto de "mayanización" (Bastos y Cumes, 2007). Es así como asistimos actualmente a unas emergencias culturales en tanto cada proceso de los mencionados sucede dentro de una tendencia mundial. Es un movimiento que puede incluirse en la llamada globalización, en tanto "lo étnico" emerge como fenómeno de moda cultural. De allí el concepto de emergencia.

Para José Bengoa (2000), es principalmente en el campo cultural donde la globalización ha estado influyendo en procesos de reconfiguración de identidades; dicha influencia puede observarse en la adopción de símbolos y modos de conducta transnacionales, así como en los cambios al interior de los pueblos indígenas. Las diferentes expresiones de las indianidades son conocidas mutuamente y circulan por los medios de la cultura dominante. Después regresan a cada espacio local, de manera que los discursos "tradicionales" cambian e incluso son reemplazados. Ocurre entonces un proceso concomitante entre globalización y particularismo (P. 30- 40). "El proceso de globalización actual es el mayor productor de nuevos discursos de identidad, en todas partes del mundo y, en particular, en América Latina" (P. 40).

Bengoa propone la teoría de la emergencia indígena en América Latina desde esta globalización del espíritu de reinventar las identidades ancestrales:

La característica principal de la emergencia indígena es la existencia de un nuevo discurso identitario, esto es, una 'cultura indígena reinventada'. Se trata de una 'lectura urbana' de la tradición indígena, realizada por los propios indígenas, en función de los intereses y objetivos indígenas (Bengoa, 2000: 128).

El proceso de etnogénesis, el discurso híbrido, el etnoecologismo, la ecoetnicidad y, en últimas, el panindigenismo, son algunos de los elementos que han jugado de manera decisiva a favor de las reinvenciones identitarias indígenas; es, en últimas, el mundo indígena globalizado ( $p$. 128-143). Guatemala es un país que ha asistido a esta movilización por lo menos durante los últimos quince 
años. De allí que en los últimos cuatro años se ha venido analizando el mismo como un proceso de mayanización (Bastos y Cumes, 2007).

Puede que en este contexto de emergencias culturales, el chaaj sea percibido como un juego que no es juego, ya que un personaje desprevenido lo podría percibir como performance, dados los elementos reunidos allí: indumentarias, pinturas corporales, ofrendas y ceremonia. Obviamente, esto no sucede para todas las personas. Si bien al actuar como espectadora, una persona se margina de los elementos simbólicos del juego en sí, ello no es óbice para que participe desde su esfera lúdica-agonal, por ejemplo, al apostar por quién logrará ganar. Puede haber alguien para quien la ceremonia previa al desarrollo del juego con la pelota merezca más que un sencillo aplauso y considere la evocación ancestral que allí se realiza.

\section{Juegos ancestrales y las desiguales emergencias culturales}

Los juegos ancestrales son constituidos por aspectos rituales, ceremoniales, lúdicos, deportivos, recreativos e históricos. Son legados que las comunidades indígenas practican actualmente. Los juegos de pelota mesoamericanos han venido siendo estudiados desde perspectivas arqueológicas e históricas, pero también practicados hasta la actualidad entre Norte y Mesoamérica. El chaaj es la variante actual más jugada en Guatemala, lo que ofrece opciones de innovación en varios aspectos de las sociedades indígenas y no-indígenas actuales. Allí son evocados los testimonios dejados por ancestras y ancestros en las estelas ${ }^{10}$ diseminadas por varios sitios arqueológicos. En el chaaj se ponen en juego los códices y las interpretaciones arqueológicas.

Pero en el chaaj también se practican los conocimientos de personas sabedoras de las comunidades; allí se reúnen los elementos culturales, deportivos y pedagógicos contemporáneos. Muestra de ello es el dinamismo del juego en algunas comunidades y aldeas de Guatemala, y los estados de Michoacán, Sonora y Sinaloa, en la república de México. En estas regiones son diversas las formas de participación de jóvenes que intercambian sus experiencias en la práctica de diferentes modalidades de la pelota mesoamericana: ulama, chaaj, tlachtli, (DIGEF, 2010: 47- 50). Fruto de esta

10 Monumentos tallados en altas piedras por las generaciones indígenas mayas que nos antecedieron. Allí dejaron plasmados varios textos en forma de glifos que datan desde alrededor del año 400 A.C. dinámica se ha venido suscitando un debate en Guatemala acerca de la pertinencia de abrir el chaaj hacia escenarios de práctica deportiva y sobre sus usos exóticos. Frente a este panorama, ¿qué tratamiento ha de darse a las innovaciones técnicas y pedagógicas con el fin de difundir la práctica del chaaj? ¿Cuál es el papel de la educación física en las comunidades indígenas con relación a su práctica? ¿Cómo aprovechar la dimensión económica de este juego ancestral, sin instrumentalizarlo como show?

En el aprovechamiento o desaprovechamiento de las manifestaciones ancestrales existen grandes desigualdades sociales y económicas. El origen de tal situación se remonta al nacimiento de las repúblicas americanas a principios del siglo XIX. En aquellas épocas muchas de las expresiones étnicas pasaron a conformar los marcadores identitarios nacionales. A través de procesos de folclorización, cada nación apropió manifestaciones ancestrales y las proyectó como imagen de identidad. Similar destino fue endosado a algunos juegos de los pueblos indígenas, los cuales entraron a hacer parte del menú nacionalista. También fueron hábilmente introducidos como prácticas deportivas en las regiones, con el fin de organizar federaciones y competencias. El zepcuagoscua es ejemplo de ello para Colombia. Similar destino está gestándose actualmente para el chaaj en Guatemala, al mejor estilo de una instrumentalización para crear identidad y percibir las naciones como "comunidades imaginadas" (Anderson, 1991).

Sin embargo, desde su dimensión sagrada, el chaaj cuestiona la instrumentalización que el mercado del turismo ha pretendido darle mediante sus hoteles, shows, espectáculos coreográficos y re-creaciones teatrales esotéricas. Como práctica lúdica, recreativa y deportiva el chaaj implica un encuentro con el cuerpo ancestral, pero también con otros conocimientos que se alimentan mutuamente. Hay deportes convencionales que al encontrarse con esta propuesta lúdica ancestral formulan interesantes innovaciones técnicas.

Ampliar la mirada más allá del juego de pelota maya como potencial de exotismo para el turismo es una de nuestras apuestas. Buscamos revisar el empleo de puestas en escena en hoteles y sitios de diversión para turistas, lo cual es sin duda otra alternativa y oportunidad para el mundo mesoamericano actual. Pero hemos de trascender esta utilización como opción económica, para manifestarlo como vehículo de conocimiento y de aprendizaje que ha permitido la pervivencia de los pueblos mayas a pesar de los siglos de violencia y exclusión. 
Conclusiones y proyecciones del chaaj como alternativa, oportunidad e innovación.

El reto que tienen las iniciativas de re-significación de juegos ancestrales es ver más allá del juego en su versión deportiva o como potencial de exotismo para el turismo. En esta perspectiva, apuntamos hacia la matriz cultural mesoamericana, que se traduce a varios idiomas mayas como Chemb'il (Mendizábal, 2010: 10-20). Esta matriz ha sido atacada por una y otra "colonización". La manipulación de las manifestaciones culturales de los pueblos con el fin de crear nación e imaginar comunidades es otra colonización; esta vez adelantada por las multinacionales y empresas que utilizan el interés global por el ecologismo y la supuesta característica indígena que le relaciona con el cuidado de la naturaleza. Responde esta realidad al argumento propuesto como la teoría del "proceso etnofágico en el imperio” (Díaz Polanco, 2006: 156-171).

Los relatos de los escritos prehispánicos nos ubican en reflexiones actuales, en la medida que expresan una conexión de eventos lúdicos, donde más que una primacía de los gestos del juego, nos reflejamos como homo ludens, en el sentido propuesto por Huizinga (1987). Entonces, los esfuerzos por reinventar prácticas ancestrales en el contexto de emergencias culturales actuales ha de considerar todos los elementos legados desde tiempos inmemoriales, pero también recoger las innovaciones de cada tiempo.

\section{Referencias}

Aj Tzuk(2011) Página oficial del grupo Aj Tzuk en facebook. Consultado el 3 de noviembre de 2011 en: http://eses.facebook.com/people/Aj-Tzuk/100000483984064

Anderson, B. (1991) [1983]. Imagined Communities: Reflections on the Origin and Spread of Nationalism (2da. edición). London and New York: Verso.

Arte Maya Naif (2011) El juego de pelota maya. Consultado el 10 de marzo de 2011 en:

http://artemayanaif.artetzutujil.com/juego-de-pelotamaya/el-juego-de-pelota-maya/

Bastos, S. y Aura C. (Coordinadores). (2007) Mayanización y vida cotidiana. La ideología multicultural en la sociedad guatemalteca. (3 Volúmenes y 1 CD). Guatemala: FLACSO, CIRMA, Cholsamaj, 2007, 1 a. edición

Bengoa, J. (2000) La emergencia indígena en América Latina. Santiago de Chile: Fondo de Cultura Económica. Chávez, A. I. (2008) [1979]. Pop Wuj / Versión de Adrian
Inés Chávez; acuarelas de Diego Rivera. México: Centro de Investigaciones y Estudios Superiores en Antropología Social: Instituto Nacional de Antropología e Historia. Fundación Diego Rivera.

Díaz-Polanco, H. (2006) Elogio de la diversidad, multiculturalismo y etnofagia. México: Siglo XXI.

DIGEF (2010). Chaaj. Los ancestrales juegos de pelota maya. Orígenes, significado y orientaciones metodológicas para su enseñanza- aprendizaje en el sistema educativo nacional. Gobierno de Guatemala, Ministerio de Educación. Dirección General de Educación Física (DIGEF). Documento disponible en: http://digef.edu.gt/biblioteca/pages/libros/Pelota_ Maya.pdf.

Huizinga, J. (1987). Homo Ludens. Madrid: Alianza Editorial. $1^{\mathrm{a}}$ ed., $2^{\mathrm{a}}$ reimpr.

Mendizábal, Oscar (2010). Un juego que no era juego. Consultado el 2 de julio de 2011 en:

http://cienporcientochapin.blogspot.com/2010/12/unjuego-que-no-era-un-juego-video.html

Mendizabal, S. (2010) El Kaba'wil en nuestra historia. Guatemala: Universidad Rafael Landívar.

MICUDE (2011) Juego de pelota maya llega a los departamentos. Recuperado el 14 de julio de 2011 en:

http://www.mcd.gob.gt/2011/05/19/juego-de-pelotamaya-llega-a-los-departamentos/

MICUDE (2008) Ministerio de cultura y deportes promueve juego de pelota. Video consultado el 13 de mayo de 2010 en: http://www.youtube.com/watch?v=8LKacPyYj7g

Panqueba, J. (2010) Muiskanoba: contemplar- describiraprehender- compartir. Sangre del alma muiska. Consultado el 2 de septiembre de 2011 en:

http://seinijsuca.blogspot.com/search/label/Chaaj

Saravia, A. (2004) [1965]. Popol Wuj. Antiguas historias de los indios Quichés de Guatemala. México: Porrúa.

------- (1969) [1965]. Popol Wuj. Antiguas historias de los indios Quichés de Guatemala. México: Porrúa. Cuarta edición.

Unesco (s.f) La tradición del teatro bailado Rabinal Achí. Consultado el 13 de julio de 2011 en: http://www. unesco.org/culture/ich/es/RL/00144.

Los Angeles Times (2010) Mayas mastered rubber long before Goodyear. Consultado el 10 de julio de 2010 en: http://articles.latimes.com/2010/may/31/science/ la-sci-rubber-20100531

Weisz, G. (1993) [1986]. El juego viviente. México: Siglo XXI. 